\title{
Targeted Therapy of Advanced Gallbladder Cancer and Cholangiocarcinoma with Aggressive Biology: Eliciting Early Response Signals from Phase 1 trials
}

Ishwaria M. Subbiah ${ }^{1}$, Vivek Subbiah ${ }^{1}$, Apostolia M. Tsimberidou ${ }^{1}$, Aung Naing ${ }^{1}$, Ahmed O. Kaseb ${ }^{2}$, Milind Javle ${ }^{2}$, Siqing Fu ${ }^{1}$, David S. Hong ${ }^{1}$, Sarina Piha-Paul ${ }^{1}$, Jennifer J. Wheler ${ }^{1}$, Kenneth R. Hess ${ }^{3}$, Filip Janku${ }^{1}$, Gerald S. Falchook ${ }^{1}$, Robert A Wolff ${ }^{1,2}$ and Razelle Kurzrock ${ }^{1,4}$

\footnotetext{
1 Department of Investigational Cancer Therapeutics,

2 Department of Gastrointestinal Medical Oncology, Division of Cancer Medicine,

${ }^{3}$ Department of Biostatistics, The University of Texas MD Anderson Cancer Center, Houston, TX

${ }^{4}$ Moores Cancer Center, University of California, San Diego, California.

Correspondence to: Ishwaria M. Subbiah, email: isubbiah@mdanderson.org
}

Keywords: gallbladder carcinoma, cholangiocarcinoma, phase l, targeted therapy, locoregional therapy

Received: January 20,2013, $\quad$ Accepted: January 26, 2013, $\quad$ Published: January 27, 2013

Copyright: @ Subbiah et al. This is an open-access article distributed under the terms of the Creative Commons Attribution License, which permits unrestricted use, distribution, and reproduction in any medium, provided the original author and source are credited.

\section{ABSTRACT:}

Purpose: Patients with advanced cholangiocarcinoma (CC) and gallbladder carcinoma (GC) have few therapeutic options for relapsed disease.

Methods: Given the overall poor prognosis in this population and the availability of novel targeted therapies, we systematically analyzed the characteristics and outcomes for GC and CC patients treated on phase I trials with an emphasis on targeted agents and locoregional therapies.

Results: Of 40 treated patients (GC=6; CC=34; median age, 60 years), $8(20 \%)$ had stable disease (SD) > 6 months, $3(8 \%)$ partial response (PR), on protocols with hepatic arterial drug infusion and anti-angiogenic, anti-HER-2/neu or novel MAPK/ ERK kinase (MEK) inhibitors. Median progression-free survival (PFS) on phase I trials was 2.0 months $(95 \%$ CI $1.7,2.8)$ versus 3.0 months ( $95 \%$ CI $2.4,5.0)$, 3.0 months (95\% CI 2.3, 4.6), and 3.0 months (95\% CI 2.4, 3.9) for their first-, second-, and last-line FDA-approved therapy. In univariate analysis, $>3$ metastatic sites, elevated alanine aminotransferase (ALT) $(>56 \mathrm{IU} / \mathrm{L})$, serum creatinine $(>1.6 \mathrm{mg} / \mathrm{dL})$, and CA19-9 $(>35 \mathrm{U} / \mathrm{mL})$ were associated with a shorter PFS. Mutational analysis revealed mutation in the KRAS oncogene in 2 of 11 patients $(18 \%)$. The SD >6 months/PR rate of $\mathbf{2 8 \%}$ was seen with hepatic arterial infusion of oxaliplatin, and inhibitors of angiogenesis, HER-2/neu or MEK.

Conclusions: The PFS in phase I trials was similar to that of the first, second, and last-line therapy $(P=0.95,0.98,0.76$, respectively) with FDA-approved agents given in the advanced setting, emphasizing a role for targeted agents in a clinical trials setting as potentially valuable therapeutic options for these patients.

\section{INTRODUCTION}

Cholangiocarcinoma (CC) and gallbladder carcinoma (GC) are rare, but aggressive, biliary tract malignancies characterized by their advanced stage at diagnosis and high mortality. Though anatomically neighbors, GC and CC are histologically distinct entities arising from the epithelium of the gallbladder and hepatic bile ducts, respectively.[1] CC is further stratified by anatomic location, either intrahepatic or extrahepatic. $\mathrm{CC}$ and $\mathrm{GC}$ are challenging diseases to diagnose given their insidious evolution and lack of effective screening modalities. With few cases $(<10 \%)$ presenting as early disease, most patients are initially diagnosed with 
Table 1: Patient Characteristics.

\begin{tabular}{|c|c|c|}
\hline & $\mathbf{N}$ & $\%$ \\
\hline \multicolumn{3}{|l|}{ Sex } \\
\hline Male & 17 & $42.5 \%$ \\
\hline Female & 23 & $57.5 \%$ \\
\hline \multicolumn{3}{|l|}{ Age at time of diagnosis, } \\
\hline Median (range) & 60.2 & $(21.6-73.6)$ \\
\hline Age $>60$ & 21 & $52.5 \%$ \\
\hline Age $<60$ & 19 & $47.5 \%$ \\
\hline \multicolumn{3}{|l|}{ Race } \\
\hline White & 31 & $77.5 \%$ \\
\hline African American & 5 & $12.5 \%$ \\
\hline Hispanic & 4 & $10.0 \%$ \\
\hline \multicolumn{3}{|l|}{ ECOG Performance Status } \\
\hline $0-1$ & 36 & $90.0 \%$ \\
\hline $2-3$ & 4 & $10.0 \%$ \\
\hline \multicolumn{3}{|l|}{ Tumor histology } \\
\hline Cholangiocarcinoma & 34 & $85.0 \%$ \\
\hline Intrahepatic & 30 & \\
\hline Extrahepatic & 4 & \\
\hline $\begin{array}{l}\text { Gallbladder } \\
\text { carcinoma }\end{array}$ & 6 & $15.0 \%$ \\
\hline \multicolumn{3}{|l|}{ Number of metastatic sites } \\
\hline Median (range) & 3 & $0-6$ \\
\hline $0-2$ & 10 & $25.0 \%$ \\
\hline$>3$ & 30 & $75.0 \%$ \\
\hline \multicolumn{3}{|l|}{ Metastatic sites } \\
\hline Liver & 36 & $90.0 \%$ \\
\hline Lymph nodes & 33 & $82.5 \%$ \\
\hline Peritoneum & 15 & $40.0 \%$ \\
\hline Lung & 15 & $37.5 \%$ \\
\hline Retroperitoneum & 15 & $37.5 \%$ \\
\hline Bone & 6 & $15.0 \%$ \\
\hline Pancreas & 5 & $12.8 \%$ \\
\hline Spleen & 4 & $10.0 \%$ \\
\hline Kidney & 1 & $2.5 \%$ \\
\hline Ovary & 1 & $2.5 \%$ \\
\hline Adrenal & 1 & $2.5 \%$ \\
\hline \multicolumn{3}{|l|}{ Prior treatments } \\
\hline $\begin{array}{ll}\text { Prior } & \text { surgical } \\
\text { resection } & \\
\end{array}$ & 15 & $37.5 \%$ \\
\hline $\begin{array}{l}\text { Prior } \\
\text { chemoembolization }\end{array}$ & 3 & $7.5 \%$ \\
\hline Prior radiation & 13 & $32.5 \%$ \\
\hline \multicolumn{3}{|c|}{ Number of prior systemic therapies } \\
\hline Median (range) & 3 & $(0-11)$ \\
\hline $0-5$ & 31 & $77.5 \%$ \\
\hline$>5$ & 9 & $22.5 \%$ \\
\hline
\end{tabular}

metastatic disease that is not amenable to surgery.[2] Despite advancements in the multimodal approach to the treatment of advanced solid tumors, the prognosis of advanced CC and GC remains grim.[2-4] Unfortunately, epidemiological trends point towards an increasing incidence and prevalence of CC and GC in East Asia, Latin America and in western societies, particularly given their association with hepatitis $\mathrm{C}$ infection, chronic nonalcoholic fatty liver disease and obesity.[5, 6] Current Food and Drug Administration (FDA) approved agents used in unresectable $\mathrm{CC}$ and GC include gemcitabine, capecitabine, cisplatin, oxaliplatin, fluoropyrimidines, including 5-fluorouracil, or a combination of these agents, although none are FDA approved primarily for use in biliary tract cancers. [7, 8] Recent protocols combine chemotherapy with radiation either in the preoperative neoadjuvant setting or in the postoperative setting. Even multi-agent chemotherapeutic approaches do not confer a durable benefit in patients with metastatic and/or relapsed disease, and fewer than $5 \%$ of patients survive 5 years after advanced diagnosis.[9]

We embarked on this study to analyze the characteristics and outcomes of patients with advanced CC and GC with very aggressive biology referred for phase I clinical trials at a major cancer center with an emphasis on targeted agents to elicit early response signals.

\section{PATIENTS AND METHODS}

We reviewed the characteristics and outcomes of 72 consecutive patients with GC and CC referred to the Clinical Center for Targeted Therapy (Phase I Clinical Trials Program) at MD Anderson Cancer Center starting from November 2004. Eligibility criteria for participation in phase I clinical trials included age $>18$ years, presence of metastatic or unresectable disease, measurable disease per Response Evaluation Criteria in Solid Tumors (RECIST) 1.0,[10] Eastern Cooperative Oncology Group (ECOG) performance status (PS) of 0-1,[11] and a life expectancy $>3$ months. Premenopausal women were required to have a negative pregnancy test and patients of childbearing potential to use contraception. Further eligibility criteria varied according to the particular study and all patients gave informed consent. This study and all clinical trials were approved by the MD Anderson Institutional Review Board.

After review of baseline clinical, laboratory, radiologic and pathologic data during the initial consultation, patients were enrolled on a phase I trial based on scientific rationale and protocol availability. After initiation of an investigational therapy, patients were evaluated at 2- to 4- week intervals, based on the specific protocol, with a medical history, physical examination, comprehensive series of laboratory tests, and assessments of toxicity and compliance. Restaging scans were done every 6-8 weeks, depending on the protocol. Obstructive cholestasis were managed according to the patients performance status and clinical status by interventional radiologist intervention or a stent placement by the gastrointestinal consult team. 


\section{Endpoints and Statistical Methods}

All statistical analysis was performed by our biostatistician (K.R.H.). Descriptive statistics summarized the patients' characteristics. Cox proportional hazards regression analysis was used to examine the association between progression-free survival (PFS) since beginning phase I therapy and the following variables measured at the time of initial phase I consultation: age at diagnosis, gender, ECOG PS, number of metastatic sites, number of prior therapies, hemoglobin, lactate dehydrogenase (LDH), platelet count, history of thromboembolism, total bilirubin, aspartate aminotransferase (AST), alanine aminotransferase (ALT), alkaline phosphatase, serum sodium, serum creatinine, tumor markers (carcinoembryonic antigen [CEA], carbohydrate antigens [CAs] CA 19-9, CA 125, CA 27.29), presence of ascites, serum albumin, and the Royal Marsden Hospital (RMH) prognostic score (comprised of serum albumin $<3.5 \mathrm{~g} / \mathrm{dL}$, lactate dehydrogenase $>$ upper limit of normal and $>2$ metastatic sites).[12]

Best response was assessed using RECIST 1.0 every 2 cycles (6-8 weeks) of therapy as per the protocol.[10]
Partial response (PR) was defined as a $>30 \%$ decrease in the sum of the longest diameter of target lesions, excluding complete disappearance of disease (complete response, $\mathrm{CR}$ ) and progressive disease (PD) was a $>20 \%$ increase. Stable disease (SD) was defined as changes that did not meet the criteria for a PR or PD. Waterfall plot analysis according to RECIST is used to illustrate response.

Overall survival was measured from the date of enrollment on a phase I trial until death from any cause or date of last follow-up. PFS is defined as the time from first day of treatment on a phase I trial to date when treatment on the last phase I trial ceased due to disease progression or death, but not toxicity. Patients still alive (in case of survival analysis) and free of progression (in case of PFS analysis) were censored at time of last followup. Toxicities were assessed using the National Cancer Institute Common Terminology Criteria for Adverse Events, version per the protocol.[13] A $P$-value $<.05$ was considered statistically significant. Statistical analysis was performed using S-PLUS ${ }^{\circledR} 8.0$ for Windows (Insightful Corp.).

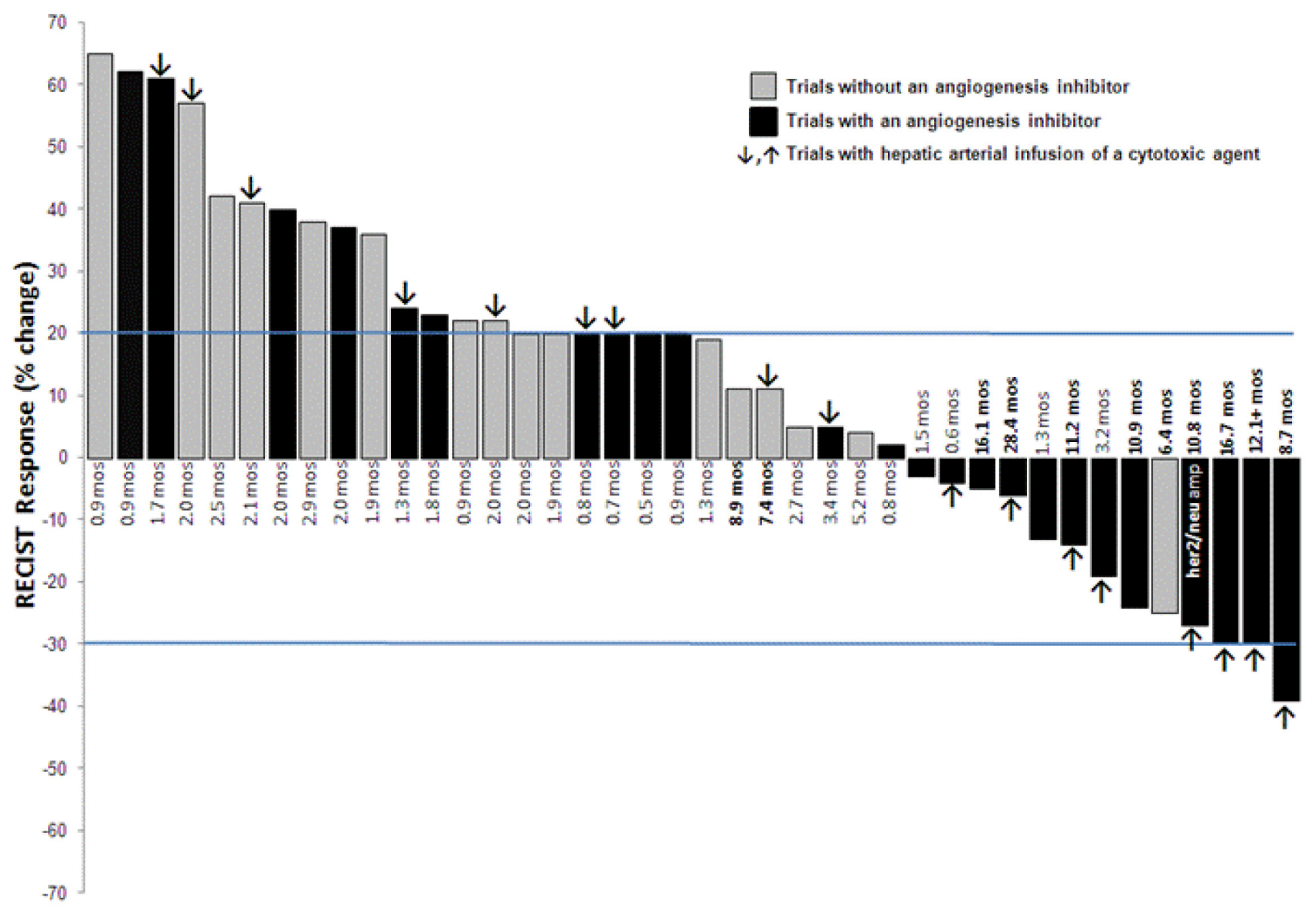

Figure 1: Waterfall plot of the best RECIST response to the best phase I trial of all $\mathbf{4 0}$ treated patients. Six patients who did not undergo restaging due to early disease progression are reflected as a $20 \%$ increase. Time (in months) reflects the duration of response. 


\section{Patients Characteristics}

Of the 72 patients with $\mathrm{GC}$ and $\mathrm{CC}$ referred to the Phase I Clinical Trials program, 32 patients $(30 \mathrm{CC}$ and 2 GC) were not enrolled in a phase I clinical trial due to deterioration of performance status $(N=25)$, decision to pursue alternate therapies including treatments closer to home $(N=6)$, and insurance denial $(N=1)$. Forty patients who participated in a phase I trial are included in this analysis. Pretreatment characteristics at presentation to the Phase I Clinic are summarized in Table 1. The median age at diagnosis was 60 years (range, 41.4-73.6 years). There were 23 women and 17 men. Thirty-one (78\%) were White, 5 (12\%) were African American, and $4(10 \%)$ were Hispanic. Seven patients (18\%) had an ECOG PS of 0,29 patients $(72 \%)$ had a PS of 1 and $4(10 \%)$ patients a 2 . Of the 40 patients treated, 6 had gallbladder carcinoma, 4 had extrahepatic cholangiocarcinoma and 30 had intrahepatic cholangiocarcinoma; the tumor histology for all 40 patients was consistent with adenocarcinoma. The median number of metastatic sites was 3 (range 0-6). The most common sites of metastases at time of phase I referral were liver ( $90 \%$ of patients), lymph nodes $(83 \%)$, peritoneum $(40 \%)$, retroperitoneum (38\%) and lung (38\%). The median time from initial phase I consultation to day one on a phase I trial was 16 days.

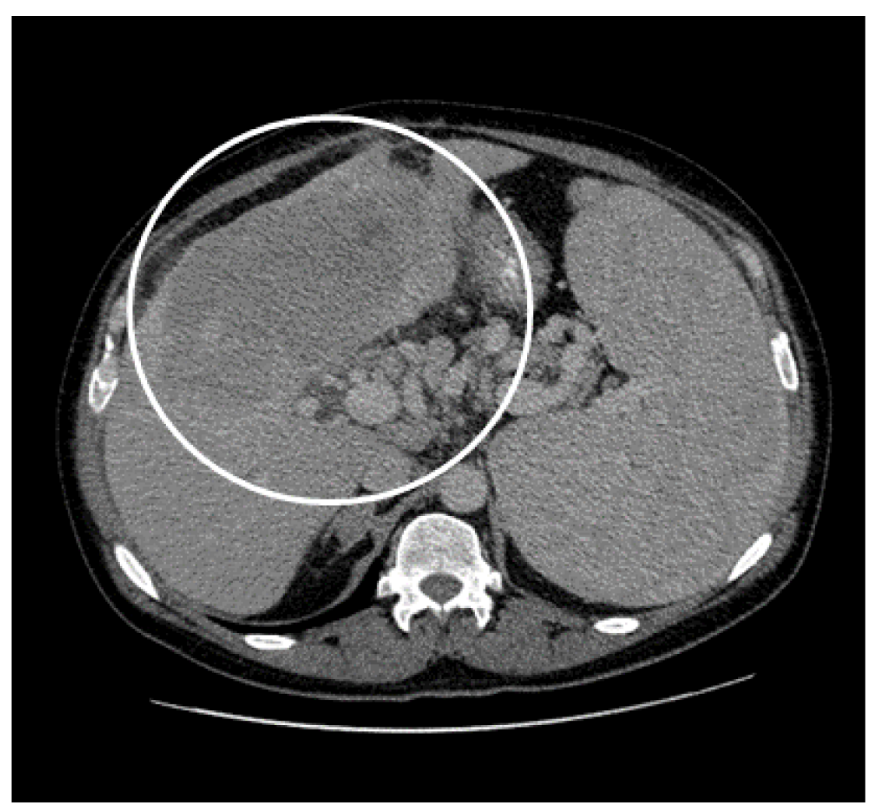

Overall, of the 40 patients enrolled on a phase I trial, two patients had received no prior therapy because of the unavailability of reasonable, conventional therapy for the extent of their disease. The remaining 38 patients had a median of 3 prior systemic therapies before referral to the Phase I Clinic (range, $1-11$ ). Fifteen patients $(38 \%)$ also underwent a prior surgical resection, whereas $13(33 \%)$ received prior radiation and $3(8 \%)$ had prior chemoembolization.

The National Comprehensive Cancer Network (NCCN) guidelines for unresectable gallbladder and cholangiocarcinoma include a gemcitabine/cisplatin combination therapy, fluoropyrimidine-based or othergemcitabine based regimen.[4, 14] For their first-line treatment in the advanced/metastatic setting, 4 of 38 patients received experimental therapy on phase II trials with a novel paclitaxel conjugate or a campotothecin analog. The remaining 34 patients received a firstline regimen based on gemcitabine $(N=24,71 \%)$, a fluoropyrimidine $(N=7,21 \%)$, or single-agent sorafenib $(N=3,8 \%)$. As their second-line therapy in the advanced disease setting, 36 patients received a regimen including gemcitabine ( $N=17,47 \%)$, a fluropyrimidine $(N=16,45 \%)$, or a targeted agent $(N=3,8 \%) ; 2$ patients did not received a second line treatment and instead began phase I therapy. All 38 patients were treated on a regimen based on FDAapproved drugs as their last antineoplastic therapy before beginning a phase I trial; $58 \%$ received a fluoropyrimidinebased regimen ( $26 \%$ received fluoropyrimidine agents in combination with platinum), whereas $23 \%$ received a

Figure 2: Prolonged partial response for $\mathbf{1 2 . 1}$ months noted in the primary hepatic lesion of a patient with advanced cholangiocarcinoma treated with hepatic arterial infusion of a cytotoxic agent along with intravenous anti-angiogenic agent. CT scan of the abdomen showed liver metastases at baseline and after 12 months of treatment. White arrows show multiple areas of hypoattenuation secondary to splenic embolization.

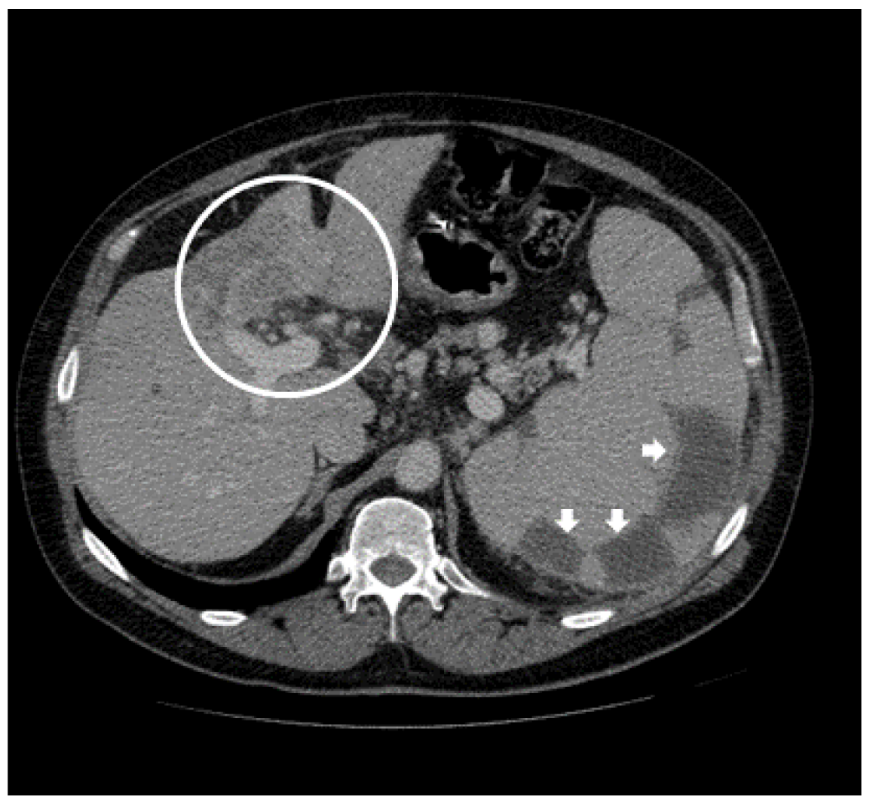


Table 2: Univariate Proportional Hazards Regression Model for Phase I Progression Free Survival (PFS)

\begin{tabular}{|c|c|c|c|c|}
\hline & $\mathbf{N}$ & \begin{tabular}{|l|} 
Median \\
PFS (mos) \\
\end{tabular} & Hazard ratio & P-value \\
\hline Gender & & & $0.5(0.3,2.1)$ & \\
\hline Male : Female & $17: 23$ & $2.9: 2.5$ & & 0.75 \\
\hline Age (yrs) & & & $0.7(0.3,1.8)$ & 0.5 \\
\hline$<60:>60$ & $19: 21$ & $2.5: 2.1$ & & \\
\hline ECOG PS & & & $2.2(0.6,7.5)$ & 0.26 \\
\hline PS $0-1: 2$ & $36: 4$ & $2.5: 1.4$ & & \\
\hline Number of metastatic site & & & $3.2(0.7,1.4)$ & 0.07 \\
\hline $1-2:>3$ & $10: 30$ & NR : 2.0 & & \\
\hline Number of prior therapies & & & $0.9(0.3,2.5)$ & 0.82 \\
\hline$<5:>5$ & $31: 9$ & $2.1: 2.9$ & & \\
\hline Hemoglobin (g/dL) & & & $1.7(0.7,4.0)$ & 0.26 \\
\hline$<10.5:>10.5$ & $25: 15$ & NR $: 2.0$ & & \\
\hline $\mathrm{LDH}(\mathrm{IU} / \mathrm{L})$ & & & $1.0(0.4,2.5)$ & 0.99 \\
\hline$<618:>618$ & $27: 13$ & $2.5: 2.0$ & & \\
\hline Platelet count (K/UL) & & & $\mathrm{n} / \mathrm{a}$ & $\mathrm{n} / \mathrm{a}$ \\
\hline$<439:>439$ & $39: 1$ & $2.5: \mathrm{NR}$ & & \\
\hline History of thromboembolism & & & $1.1(0.4,3.0)$ & 0.86 \\
\hline Yes : No & $11: 29$ & $2.5: 2.0$ & & \\
\hline Total bilirubin (mg/dL) & & & $1.6(0.6,4.2)$ & 0.33 \\
\hline$<1:>1$ & $30: 10$ & $2.9: 2.0$ & & \\
\hline ALT (IU/L) & & & $5.3(1.8,16)$ & 0.0072 \\
\hline$<56:>56$ & $35: 5$ & NR : 1.3 & & \\
\hline AST (IU/L) & & & $1.0(0.4,2.3)$ & 0.91 \\
\hline$<46:>46$ & $21: 19$ & $2.1: 2.9$ & & \\
\hline Alkaline phosphatase (IU/L) & & & $0.6(0.2,1.5)$ & 0.3 \\
\hline$<126:>126$ & $12: 28$ & $2.0: 2.9$ & & \\
\hline Serum sodium $(\mathrm{mEq} / \mathrm{L})$ & & & $1.4(0.4,4.6)$ & 0.64 \\
\hline$>135:<135$ & $36: 4$ & $2.1: 2.4$ & & \\
\hline Serum creatinine $(\mathrm{mg} / \mathrm{dL})$ & & & $\mathrm{n} / \mathrm{a}$ & 0.022 \\
\hline$<1.6:>1.6$ & $36: 4$ & $2: \mathrm{NR}$ & & \\
\hline CEA (ng/mL) & & & $1.2(0.4,3.8)$ & 0.69 \\
\hline$<6:>6$ & $33: 7$ & $2.5: 1.8$ & & \\
\hline CA-19-9 (U/ml) & & & $4.8(1.1,21)$ & 0.01 \\
\hline$<35:>35$ & $11: 29$ & NR : 2.0 & & \\
\hline CA $125(\mathrm{U} / \mathrm{ml})$ & & & $1.1(0.4,2.6)$ & 0.87 \\
\hline$<35:>35$ & $20: 20$ & $2.0: 2.9$ & & \\
\hline CA $27.29(\mathrm{U} / \mathrm{ml})$ & & & $1.2(0.5,3.0)$ & 0.66 \\
\hline$<47:>47$ & $22: 16$ & $2.5: 2.0$ & & \\
\hline Presence of ascites & & & $1.1(0.5,2.7)$ & 0.79 \\
\hline Yes : No & $19: 21$ & $2.1: 2.9$ & & \\
\hline Serum albumin $(g / d L)$ & & & $1.5(0.6,3.8)$ & 0.44 \\
\hline$<3.5:>3.5$ & $29: 11$ & $2.5: 2.0$ & & \\
\hline RMH score & & & $1.5(0.6,3.7)$ & 0.34 \\
\hline $0-1: 2-3$ & $21: 19$ & $2.9: 2.0$ & & \\
\hline
\end{tabular}

Abbreviations: ALT alanine aminotransferase; AST aspartate aminotransferase; ECOG, Eastern Cooperative Oncology Group; LDH lactate dehydrogenase; CEA Carcinoembryonic antigen; CA carbohydrate antigen; NR not reached; PS, performance status; RMH Royal Marsden Hospital; n/a Not applicable 
targeted agent, most commonly an angiogenesis inhibitor. Thus FDA-approved agents formed the backbone of the systemic combination therapy prior to enrollment in a phase I trial.

\section{Treatment}

Overall, patients were initially treated on 1 of 22 phase I clinical trials; of these trials, 8 patients received therapy on six first-in-human trials with novel targeted inhibitors against MEK, VEGF, gamma-secretase, aurora kinase and the IGF-IR pathway. Of 40 patients, $30(75 \%)$ were treated on a trial with combination therapy of two or more agents while 10 patients $(25 \%)$ received treatment with a single agent. Seventeen (43\%) patients received locoregional treatment with direct infusion of a cytotoxic agent into the hepatic artery; 24 $(60 \%)$ received intravenous angiogenesis inhibitors, of whom $13(33 \%)$ received the antiangiogenic agent in combination with hepatic arterial infusion therapy. Fifteen $(38 \%)$ patients received targeted agent(s) alone, $20(50 \%)$ received targeted agent(s) in combination with cytotoxic chemotherapy, and $5(12 \%)$ received cytotoxic chemotherapy alone. The median number of cycles received was 2 (range $1-12$ ). Six patients went on to
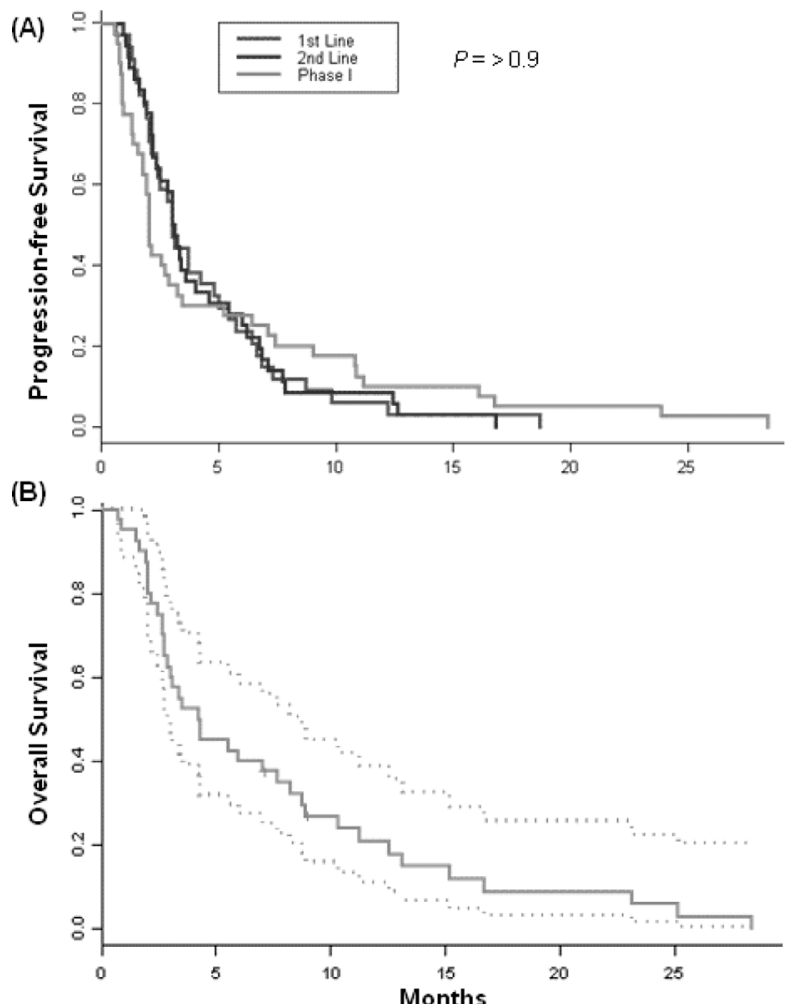

Figure 3: (A) PFS of patients treated on phase I trials compared to their first-line, second-line and last systemic antitumor therapy given in the advanced setting prior to phase I referral. (B) Median overall survival after starting a phase I trial. Dotted lines represent $95 \%$ confidence intervals for the estimated survival probabilities. receive therapy under a second phase I trial; of these patients, 3 received treatment on 3 phase I trials.

\section{Response}

Six patients were not restaged prior to end of cycle 2 due to clinical deterioration and early disease progression. Of the 40 patients treated on clinical studies, $3(8 \%)$ had a partial response (PR), 17 had stable disease (43\%) including $8(20 \%)$ who had SD $>6$ months, and 20 patients had progressive disease (PD) with a SD> 6 months/PR rate of $27.5 \%$ (Figure 1). The highest rates of prolonged $\mathrm{SD}>6$ months/PR were observed in patients treated with protocols that included hepatic arterial infusion (HAI) of oxaliplatin or paclitaxel (7 of 17 patients treated on HAI regimens, $41 \%$ ) or antiangiogenic agents ( 9 of 24 patients, 38\%) with 6 of those patients receiving treatment that included HAI of oxaliplatin combined with systemic bevacizumab (Figure 2; Table 3 ). A prolonged SD of 10.8 months was observed in a patient with metastatic GC with HER-2/neu amplification who was treated on phase I protocols, first combining the anti-HER-2/neu antibody trastuzumab and the HER2/neu tyrosine kinase inhibitor lapatinib, and then with trastuzumab and erlotinib. Prolonged SD lasting 9 months was also seen in one patient treated on a first-in-human trial using a novel MEK inhibitor.

\section{Survival and toxicities}

For the 40 patients treated on phase I trials, the time from diagnosis of advanced (metastatic) disease to primary evaluation in the Phase I Clinical Center for Targeted Therapy was 13.1 months. The median time from the initial phase I consultation to day 1 of a trial was 16 days. The overall median survival from day 1 on a phase I trial was 4.2 months (95\% CI 3.8 - 8.6 months). Median PFS for 40 treated patients was 2.0 months $(95 \%$ CI $1.8,3.4)$ on phase I clinical trials. Among these 40 patients, the median PFS on their first-line and second-line prior therapies with FDA-approved agents given in the advanced setting was 3.0 months $(95 \%$ CI $2.4,5.0)$ and 3.0 months $(95 \%$ CI $2.3,4.6)$, respectively. Median PFS on their last FDAapproved treatment before phase I referral was 3.0 months (95\% CI 2.4, 3.9). In comparison, the median PFS on phase I therapy did not differ significantly from that on their first-line or second-line FDA-approved agents given for advanced disease or their last treatment prior to phase I referral ( $P=0.95,0.98,0.76$, respectively) (Figure 3A). Data were also analyzed from the 32 patients who were not enrolled on phase I trials and the PFS of their first-, second-line, and last treatment with FDA-approved agents prior to phase I referral (3.1, 2.4, 3.0 months, respectively) also did not differ significantly from the PFS of the phase I-treated patients. 
Table 3: Characteristics of patients with clinical benefit rate (PR $+\mathrm{SD}>6$ months).

\begin{tabular}{|c|c|c|c|c|c|}
\hline $\mathbf{P t}$ & $\begin{array}{l}\text { Age/ } \\
\text { Gender }\end{array}$ & Diagnosis & Phase I Regimen(s) & Best response & $\begin{array}{|lr|}\text { PFS } & \text { on } \\
\text { Phase I trial } \\
\text { (months) }\end{array}$ \\
\hline 1 & $57 / \mathrm{F}$ & Intrahepatic CC & $\begin{array}{l}\text { HAI Oxaliplatin + IV Bevacizumab + } \\
\text { IV 5FU }\end{array}$ & $\begin{array}{l}\text { PR on Phase } \\
\text { I, NED post } \\
\text { resection** }\end{array}$ & 16.7 \\
\hline 22 & $55 / \mathrm{M}$ & Intrahepatic CC & $\begin{array}{l}\text { HAI Oxaliplatin + IV 5FU + IV } \\
\text { Bevacizumab + IV Cetuximab }\end{array}$ & PR & $9+$ \\
\hline 34 & $47 / \mathrm{F}$ & Intrahepatic CC & $\begin{array}{l}\text { HAI Nab-Paclitaxel + IV Gemcitabine } \\
+ \text { IV Bevacizumab }\end{array}$ & PR & $7.1+$ \\
\hline 8 & $55 / \mathrm{F}$ & Intrahepatic CC & $\begin{array}{l}\text { HAI Oxaliplatin + IV Bevacizumab + } \\
\text { IV 5FU }\end{array}$ & SD & 6.4 \\
\hline 37 & $69 / \mathrm{F}$ & Intrahepatic CC & $\begin{array}{l}\text { HAI Oxaliplatin + IV Bevacizumab + } \\
\text { IV 5FU }\end{array}$ & SD & 7.2 \\
\hline 26 & $71 / \mathrm{F}$ & Extrahepatic CC & HAI Nab-Paclitaxel & SD & 7.4 \\
\hline 36 & $61 / \mathrm{F}$ & $\begin{array}{l}\text { Gallbladder } \\
\text { carcinoma }\end{array}$ & $\begin{array}{l}\text { Bevacizumab }+ \text { Trastuzumab } \\
+ \text { Lapatinib; Trastuzumab + Erlotinib }\end{array}$ & SD & 10.4 \\
\hline 25 & $47 / \mathrm{M}$ & Intrahepatic CC & Bevacizumab + Sorafenib & SD & 10.9 \\
\hline 39 & 67/M & Intrahepatic CC & $\begin{array}{l}\text { HAI Oxaliplatin + IV Bevacizumab + } \\
\text { IV 5FU }\end{array}$ & SD & 11.3 \\
\hline 38 & $60 / \mathrm{F}$ & Intrahepatic CC & $\begin{array}{lcl}\text { Bevacizumab } & + & \text { Sorafenib; } \\
\text { Sirolimus }+ \text { Cetuximab } & \end{array}$ & SD & 14.3 \\
\hline 15 & $53 / \mathrm{M}$ & Intrahepatic CC & Novel MEK inhibitor & SD & 8.9 \\
\hline
\end{tabular}

5-FU fluorouracil; CC cholangiocarcinoma; CR complete response; HAI hepatic arterial infusion; PR partial response; SD stable disease

** This patient went on to receive neoadjuvant bevacizumab + proton therapy, followed by an extended hepatectomy, ultimately showing no evidence of disease (NED).

Overall survival from enrollment on a phase I trial to date of death is shown in Figure 3B. Among the 40 treated patients, $38(95 \%)$ had died at the time of analysis. The 90 -day mortality was $38 \%$ with 25 patients alive at 3 months after beginning phase I therapy; the 6-month mortality was $63 \%$ with 15 patients alive at 6 months after beginning therapy on phase I trials. Importantly, there was no treatment-related mortality. Two patients treated on different combination regimens that included an angiogenesis inhibitor experienced grade 3 gastrointestinal bleeding, prompting a change of regimen, with overall progression-free period of 10.8 and 16.8 months, respectively, on their subsequent phase I trials. The only other grade 3 toxicity that prompted removal from study was the development of an arteriovenous fistula in one patient due to the placement of the hepatic arterial infusion catheter.

\section{Prognostic Factors for Survival}

We conducted univariate analysis to evaluate the effects on survival of variables including age, sex, race/ ethnicity, ECOG performance status, tumor markers (carcinoembryonic antigen [CEA], carbohydrate antigens [CAs] CA 19-9, CA 125, CA 27.29); history of thromboembolism; number of prior therapies; presence of liver metastases; number of metastatic sites; leukocyte count; hemoglobin level; platelet count; and albumin, lactate dehydrogenase (LDH), alkaline phosphatase, bilirubin, alanine aminotransferase, aspartate aminotransferase, RMH score and serum creatinine levels (Table 2). In univariate analysis, factors associated with a shorter Phase I PFS were a higher number of metastatic sites (1-2 versus $>3$; hazard ratio [HR] 3.2, $P=0.070$ ), elevated serum ALT ( $>56 \mathrm{IU} / \mathrm{L}$; HR 5.3, $P=0.007$ ), elevated serum creatinine $(>1.6 \mathrm{mg} / \mathrm{dL} ; P=0.022)$, and high CA19-9 ( $>35 \mathrm{U} / \mathrm{mL}$; HR 4.8, $P=0.010)$.

\section{Molecular analysis}

Testing for mutations in KRAS, NRAS, BRAF, cKIT, EGFR, PIK3CA, TP53, as well as immunohistochemistry for PTEN loss and HER-2/neu FISH amplification was completed on patients with adequate available tissue in the MD Anderson CLIA-certified laboratory. DNA was extracted from micro-dissected paraffinembedded tumor and analyzed by a PCR-based DNA sequencing method to examine codons 12,13 and 61 of 
Table 4: Molecular analyses of the 40 treated patients treated on Phase I trials.

\begin{tabular}{|c|c|c|}
\hline $\begin{array}{l}\text { Molecular } \\
\text { mutation } \\
\text { aberration }\end{array}$ & $\begin{array}{l}\text { Number } \\
\text { of patients } \\
\text { tested }\end{array}$ & \begin{tabular}{|lr} 
Number & of \\
patients & with \\
a mutation or \\
aberration
\end{tabular} \\
\hline KRAS & 11 & 2 \\
\hline NRAS & 7 & 0 \\
\hline BRAF & 10 & 0 \\
\hline CKIT & 3 & 0 \\
\hline EGFR & 10 & 0 \\
\hline PI3K & 9 & 0 \\
\hline GNAQ & 1 & 0 \\
\hline PTEN Loss & 3 & 0 \\
\hline ER IHC & 6 & 0 \\
\hline PR IHC & 6 & 0 \\
\hline HER-2/neu FISH & 8 & 1 \\
\hline
\end{tabular}

Abbreviations: IHC immunohistochemistry; FISH fluorescence in situ hybridization

the KRAS proto-oncogene. Of 11 tested patients, a KRAS mutation was detected in the tumor of two patients with cholangiocarcinoma, one in codon 12 and another in codon 13.[15] A third patient's gallbladder carcinoma demonstrated FISH amplification of HER-2/neu (HER-2/ neu: CEP17 signal ratio: 6.49). This patient maintained SD for 27 months with a HER-2/neu targeting agent prior to progression; then, phase I therapy including HER-2/ neu targeting agents resulted in SD for an additional 10.8 months.

\section{DISCUSSION}

Treatment options for patients with advanced unresectable GC and $\mathrm{CC}$ are limited given the poor outcomes of even frontline therapies with FDA-approved agents. Complete surgical resection remains the only curative modality of therapy, offering benefit only for patients with localized disease. The NCCN guidelines for relapsed GC and CC highlight the limited clinical trial data that can be used to define a standard regimen or definitive benefit with an emphasis on clinical trial participation.[3, 16]

Approximately $44 \%$ of patients with CC and GC referred for phase I trials were ineligible, mainly because of poor performance status (ECOG PS 3 or greater). Our analysis shows $28 \%$ of our patients achieved SD $>6$ months/PR mainly with locoregional therapy with hepatic arterial infusion and angiogenesis inhibitors, suggesting that these modalities merit further investigation in similar patients. Additionally, the fact that SD > 6 months was seen with targeted therapies including a
MEK inhibitor (SD 9 months) and an HER-2/neu targeting agent (SD 11 months) may be of investigational interest. Indeed, Bendell and colleagues also demonstrated a PR in cholangiocarcinoma treated with a MEK $1 / 2$ inhibitor. [17] Although $K R A S$ mutations may sensitize a tumor to MEK inhibitors,[18] the $K R A S$ status of our patient with prolonged stable disease on a novel MEK inhibitor was unknown due to lack of tissue available for molecular analysis.

Overall, the pattern of molecular aberrations seen in $\mathrm{GC}$ and $\mathrm{CC}$ is slowly emerging. Somatic mutations of $K R A S$, most commonly G12D and G13D, and PIK3CA, most commonly E545K, along with deletion of $p 53$ have been reported in CCs; activating mutations in PIK3CA have also been reported in $12.5 \%$ of patients with GC, highlighting the therapeutic targeting potential of the RAS/RAF/MEK/ERK pathway and PI3K/AKT/MTOR pathway.[19, 20] It is conceivable that the responses to MEK inhibitors seen in $\mathrm{CC}$ are due to the frequency of KRAS mutations among these patients (13\%).[20] Most recently, tissue from 9 of 40 patients (23\%) with intrahepatic CC was shown to harbor mutations in the gene encoding isocitrate dehydrogenase 1 (IDH1).[21] The clinical significance of this is not known, but one proposed mechanism demonstrates that a heterozygous IDHI mutation leads to elevated levels of hypoxia-inducible factor subunit HIF-1alpha, thereby promoting tumor development and suggesting that IDH1 may be a tumor suppressor. We have recently shown that better response rates, time to treatment failure, and overall survival are associated with matching therapy to actionable mutations, highlighting the importance of further assessment of this strategy in these populations.[22]

Our analysis demonstrates that the PFS of phase I therapy in our program is comparable to that of the first-line, second-line and last prior treatment options with FDA-approved agents for advanced disease. There are several limitations to our analysis. First, a selection bias exists within our patient population given that the patients presented with widely metastatic disease and the PFS on frontline therapies in this population, particularly following treatment with the gemcitabine/ cisplatin combination, was shorter than that reported in the pivotal phase III trial.[14] Such findings may suggest a more aggressive tumor behavior that has yet to be characterized among our patients. Second, a substantial number of patients ( 32 of 72 referred patients, $44 \%$ ) were not enrolled on a phase I trial. This was due, for the most part, to clinical deterioration, although the first-, second-, and last line PFS among these untreated patients did not differ from that of the 40 patients treated on a phase I trial. Thirdly, we were unable to conduct an effective multivariate analysis given the small sample size. Finally, mutational analysis was only performed in a small subset of patients due to the lack of tissue availability for testing. Additionally, data on quality of life as an endpoint was not 
collected and, given the poor prognosis of these patients, an emphasis on quality of life measurements becomes critical. Future clinical trials should include quality of life as an important end point.

Predictors of shorter phase I PFS in univariate analysis were a higher number of metastatic site $(>3)$, elevated serum ALT ( $>56 \mathrm{IU} / \mathrm{L})$, elevated serum creatinine ( $>1.6 \mathrm{mg} / \mathrm{dL})$, and high CA19-9 (>35 U/mL). Overall, the short PFS reflects the rather dismal outcomes of these advanced $\mathrm{GC}$ and $\mathrm{CC}$ patients and emphasizes the need for new approaches. Since $28 \%$ of our patients who were able to enroll of a trial achieved SD $>6$ months/PR mainly on our trials with locoregional therapy, anti-angiogenic agents, MEK and HER-2/neu inhibitors, these strategies may merit further exploration.

\section{REFERENCE}

1. Yamaguchi K and Enjoji M. Carcinoma of the gallbladder. A clinicopathology of 103 patients and a newly proposed staging. Cancer. 1988; 62(7):1425-1432.

2. Zhu AX, Hong TS, Hezel AF and Kooby DA. Current management of gallbladder carcinoma. Oncologist. 2011; 15(2):168-181.

3. Crane $\mathrm{CH}$, Macdonald $\mathrm{KO}$, Vauthey JN, Yehuda $\mathrm{P}$, Brown T, Curley S, Wong A, Delclos M, Charnsangavej $\mathrm{C}$ and Janjan NA. Limitations of conventional doses of chemoradiation for unresectable biliary cancer. Int J Radiat Oncol Biol Phys. 2002; 53(4):969-974.

4. Hezel AF and Zhu AX. Systemic therapy for biliary tract cancers. Oncologist. 2008; 13(4):415-423.

5. Tyson GL and El-Serag HB. Risk factors for cholangiocarcinoma. Hepatology. 2011; 54(1):173-184.

6. Welzel TM, Graubard BI, El-Serag HB, Shaib YH, Hsing AW, Davila JA and McGlynn KA. Risk factors for intrahepatic and extrahepatic cholangiocarcinoma in the United States: a population-based case-control study. Clin Gastroenterol Hepatol. 2007; 5(10):1221-1228.

7. Khan SA, Davidson BR, Goldin R, Pereira SP, Rosenberg WM, Taylor-Robinson SD, Thillainayagam AV, Thomas HC, Thursz MR and Wasan H. Guidelines for the diagnosis and treatment of cholangiocarcinoma: consensus document. Gut. 2002; 51 Suppl 6:VI1-9.

8. Kondo S, Takada T, Miyazaki M, Miyakawa S, Tsukada K, Nagino M, Furuse J, Saito H, Tsuyuguchi T, Yamamoto M, Kayahara M, Kimura F, Yoshitomi H, Nozawa S, Yoshida $\mathrm{M}$, Wada K, et al. Guidelines for the management of biliary tract and ampullary carcinomas: surgical treatment. J Hepatobiliary Pancreat Surg. 2008; 15(1):41-54.

9. Anderson CD, Pinson CW, Berlin J and Chari RS. Diagnosis and treatment of cholangiocarcinoma. Oncologist. 2004; $9(1): 43-57$.

10. Therasse P, Arbuck SG, Eisenhauer EA, Wanders J, Kaplan RS, Rubinstein L, Verweij J, Van Glabbeke M, van Oosterom AT, Christian MC and Gwyther SG. New guidelines to evaluate the response to treatment in solid tumors. European Organization for Research and Treatment of Cancer, National Cancer Institute of the United States, National Cancer Institute of Canada. J Natl Cancer Inst. 2000; 92(3):205-216.

11. Oken MM, Creech RH, Tormey DC, Horton J, Davis TE, McFadden ET and Carbone PP. Toxicity and response criteria of the Eastern Cooperative Oncology Group. Am J Clin Oncol. 1982; 5(6):649-655.

12. Arkenau HT, Barriuso J, Olmos D, Ang JE, de Bono J, Judson I and Kaye S. Prospective validation of a prognostic score to improve patient selection for oncology phase I trials. J Clin Oncol. 2009; 27(16):2692-2696.

13. Trotti A, Colevas AD, Setser A, Rusch V, Jaques D, Budach V, Langer C, Murphy B, Cumberlin R, Coleman CN and Rubin P. CTCAE v3.0: development of a comprehensive grading system for the adverse effects of cancer treatment. Semin Radiat Oncol. 2003; 13(3):176-181.

14. Valle J, Wasan H, Palmer DH, Cunningham D, Anthoney A, Maraveyas A, Madhusudan S, Iveson T, Hughes S, Pereira SP, Roughton M and Bridgewater J. Cisplatin plus gemcitabine versus gemcitabine for biliary tract cancer. $\mathrm{N}$ Engl J Med. 2010; 362(14):1273-1281.

15. De Roock W, Jonker DJ, Di Nicolantonio F, SartoreBianchi A, Tu D, Siena S, Lamba S, Arena S, Frattini M, Piessevaux H, Van Cutsem E, O'Callaghan CJ, Khambata-Ford S, Zalcberg JR, Simes J, Karapetis CS, et al. Association of KRAS p.G13D mutation with outcome in patients with chemotherapy-refractory metastatic colorectal cancer treated with cetuximab. JAMA. 2010; 304(16):18121820.

16. Macdonald $\mathrm{OK}$ and Crane $\mathrm{CH}$. Palliative and postoperative radiotherapy in biliary tract cancer. Surg Oncol Clin N Am. 2002; 11(4):941-954.

17. Bendell JC PK, Jones SF, Barrett E, Guthrie K, Kass CL, Litwiler KS, Napier C and Patnaik A. (2011). A phase I dose-escalation study of MEK inhibitor MEK162 (ARRY438162 ) in patients with advanced solid tumors. AACRNCI-EORTC International Conference: Molecular Targets and Cancer Therapeutics. (San Francisco, CA.

18. Engelman JA, Chen L, Tan X, Crosby K, Guimaraes AR, Upadhyay R, Maira M, McNamara K, Perera SA, Song Y, Chirieac LR, Kaur R, Lightbown A, Simendinger J, Li T, Padera RF, et al. Effective use of PI3K and MEK inhibitors to treat mutant Kras G12D and PIK3CA H1047R murine lung cancers. Nat Med. 2008; 14(12):1351-1356.

19. Xu RF, Sun JP, Zhang SR, Zhu GS, Li LB, Liao YL, Xie JM and Liao WJ. KRAS and PIK3CA but not BRAF genes are frequently mutated in Chinese cholangiocarcinoma patients. Biomed Pharmacother. 2011; 65(1):22-26.

20. Deshpande V, Nduaguba A, Zimmerman SM, Kehoe SM, Macconaill LE, Lauwers GY, Ferrone C, Bardeesy N, Zhu AX and Hezel AF. Mutational profiling reveals PIK3CA mutations in gallbladder carcinoma. BMC Cancer. 2011; 
11:60.

21. Borger DR, Tanabe KK, Fan KC, Lopez HU, Fantin VR, Straley KS, Schenkein DP, Hezel AF, Ancukiewicz M, Liebman HM, Kwak EL, Clark JW, Ryan DP, Deshpande V, Dias-Santagata D, Ellisen LW, et al. Frequent Mutation of Isocitrate Dehydrogenase (IDH) 1 and IDH2 in Cholangiocarcinoma Identified Through Broad-Based Tumor Genotyping. Oncologist. 2011.

22. Tsimberidou AM IN, Hong DS, Wheler JJ, Fu S, PihaPaul SA, Naing A, Falchook GS, Janku F, Luthra R, Wen S, Kurzrock R. Personalized medicine in a phase I clinical trials program: The M. D. Anderson Cancer Center Initiative. J Clin Oncol. 2011 29: 2011 (suppl; abstr CRA2500). 